\title{
Characterising the plant based component of the Irish diet in terms of its micronutrient content
}

\author{
G. Kent ${ }^{1,2}$, L. Kehoe ${ }^{1}$, R. McCarthy ${ }^{1}$, B. A. McNulty ${ }^{3}$, A. P. Nugent ${ }^{4}$, A. Flynn ${ }^{1}$ and \\ J. Walton ${ }^{2}$ \\ ${ }^{1}$ School of Food and Nutritional Sciences, University College Cork, Ireland, \\ ${ }^{2}$ Dept. Biological Sciences, Cork Institute of Technology, Ireland, \\ ${ }^{3} U C D$ Institute of Food and Health, University College Dublin, Ireland and \\ ${ }^{4}$ Institute for Global Food Security, Queens University Belfast, N. Ireland
}

A diet rich in plant-based foods with fewer animal products may offer improved health and environmental benefits ${ }^{(1)}$. There is little consensus in the literature on the definition of a plant-based diet with some defining it as one rich in vegetables, legumes, fruits, wholegrains, nuts and seeds, excluding animal foods and with heavy restrictions on processed foods ${ }^{(2)}$. Other definitions make no reference to the inclusion/exclusion of processed foods and refer only to the exclusion of all animal foods from the total diet ${ }^{(3)}$. The diet (foods and beverages) of Irish adults has previously been characterised and evaluated in terms of macronutrient quality according to two extremes of plant-based diet definitions ${ }^{(4)}$. The aim of this study was to further that exploration in this cohort by examining the micronutrient quality for each definition.

Data from the Irish National Adult Nutrition Survey (NANS) (2008-2010) were used. Food intake data were collected using a 4-day semi-weighed food record from 1500 adults (18-90y). Nutrient intakes were analysed using UK and Irish food composition databases. All foods consumed in the NANS were categorised into two definitions; 1. plant-based component of the diet and 2. total diet excluding all animal components. The plant-based component included vegetables, legumes, fruits, wholegrains, nuts and seeds and excluded all animal foods and processed foods. The second categorisation included all non-animal foods regardless of processing. Nutritional quality was assessed by estimating energy-adjusted (intake/10MJ) intakes of vitamins A, E, C, thiamin, niacin, pantothenate, biotin, vitamin B6, dietary folate equivalents (DFE), potassium, calcium, iron, magnesium and phosphorous from both using SPSSC v26. Differences in intakes $(\mathrm{p}<0.01)$ were assessed using a paired sample t-test.

The plant-based component of the diet provided significantly higher $(p<0.01)$ intakes of vitamins $\mathrm{A}, \mathrm{E}, \mathrm{C}$, thiamin, riboflavin, pantothenate, biotin, vitamin B6, DFE, potassium, iron, magnesium and phosphorous compared to intakes from the total diet excluding all animal foods while intakes of calcium and niacin were similar in the two plant-based definitions. Intakes of vitamins $\mathrm{D}(1.0 \mu \mathrm{g})$ and B12 $(1.8 \mu \mathrm{g})$ were significantly lower $(\mathrm{p}<0.01)$ in the plant-based component of the diet compared to the total diet excluding all animal components $(2.2 \mu \mathrm{g}$ and $0.11 \mu \mathrm{g}$, respectively). This can be explained by the exclusion of processed foods (including fortified ready-to-eat breakfast cereals, fat spreads and beverages) from the plant-based component.

Overall, the micronutrient profile of the plant-based component of the diet was of higher nutritional quality than the total diet excluding all animal components, except for vitamins D and B12. This study highlights the variability in nutritional quality between different definitions of plant-based diets and the importance of fortified processed foods for vitamins D and B12 intake in the diets of Irish adults (in the absence of animal products).

\section{References}

1. Garton L, Hood S. (2017) British Dietetic Association (BDA) Food Fact Sheet: Plant-Based Diet.

2. Ostfeld R.J. (2017) J Geriatr Cardiol; 14:315.

3. Toumpanakis A, Turnbull T, Alba-Barba I. (2018) Open Diabetes Res. Care; 6 (1): Eooo534.

4. Kent G, Kehoe L, McCarthy R, et al. (2020) Proc Nutr Soc 79 (OCE2), E562. 